\title{
Políticas públicas: proceso de concertación Estado-Sociedad
}

\author{
Maggiolo, Isabel ${ }^{\star}$ \\ Perozo Maggiolo, Javier ${ }^{\star *}$
}

\section{Resumen}

Este artículo se propone caracterizar las políticas públicas, como proceso de concertación Estado-Sociedad, mediante el examen de la interacción entre éstos para llegar a un punto de acuerdo, las políticas públicas. Se empleó la observación de los planteamientos de Subirats (1989), Sagasti (1999), Ballart (1992), Repetto (2000), Piñango (2003) y Loaiza (2004), entre otros, en cuanto elementos teórico-conceptuales sobre políticas públicas, actores, evaluación de políticas y Estado y sociedad. Estableciéndose como principales conclusiones que las políticas públicas son el resultado de actos de confrontación/acuerdo entre representantes estatales y sociales, que enfrentan nudos críticos, situaciones conflictivas y restricciones normatizadas, donde por su naturaleza política el Estado está implícito, pero la sociedad requiere de cierto empoderamiento social y canales de comunicación efectivos que avalen una sana y estrecha relación Estado-Sociedad, en procura de obtener la aprobación pública.

Palabras clave: Política pública, Estado, sociedad, empoderamiento social.

\section{Public Policies: the Process of State-Society Agreement}

\section{Abstract}

This article proposes characterizing public policies as a process of State-Society agreement, by examining their interaction to reach a point of agreement: the public policies. The approaches of Subirats (1989), Sagasti (1999), Ballart (1992), Repetto (2000), Piñango (2003) and Loaiza (2004),

Recibido: 12-07-06. Aceptado: 23-05-07

Doctora en Ciencias Médicas, MSc en Planificación y Gerencia de Ciencia y Tecnología, Especialista en Ginecología y Obstetricia. Profesora Titular adscrita al Departamento de Obstetricia y Ginecología de la Escuela de Medicina de la Universidad Zulia.

E-mail: maggisab2@yahoo.com

** Abogado, Especialista en Tributaria. Candidato a MSc. en Gerencia Pública, Cursante regular del Doctorado en Ciencias Jurídicas de la Universidad del Zulia.

E-mail: javiersaidpm@hotmail.com 
among others, were studied for their theoretic-conceptual elements regarding public policies, actors and the evaluation of policies, State and society. Principle conclusions were that public policies are the result of acts of confrontation/agreement between state and social representatives, who face critical knots, conflicting situations and regulated restrictions, where the State is implicit due to its political nature, while society requires certain social empowerment and effective communication channels to guarantee a healthy, close State-Society relationship to obtain public approval.

Key words: Public policy, State, society, social empowerment.

\section{Introducción}

Esta investigación corresponde al área de análisis de políticas públicas, entendida dicha área, apoyándonos en Ballart $^{1}(1992: 43,44)$ como el estudio de las diferentes políticas o planes de actuación públicos en torno a sus efectos probables, estimados o proyectados.

Partiendo de la base, de que la importancia de las políticas públicas (PP), especialmente de las sociales ${ }^{2}$, radica en su relación natural con el desarrollo nacional, la problemática social, puede indagarse desde la perspectiva de la interacción entre actores sociales y estatales en torno de una necesidad que logra convertirse en tema de interés público, lo cual, permitiría deducir que toda política social, es un proceso donde convergen, individuales perspectivas sobre la realidad que se pretende cambiar o modificar.
Entonces, ¿cómo lograr una relación Estado-sociedad, que priorice el bienestar social en la hechura de PP? ¿Constituyen las PP, reales procesos de acuerdo público, Estado-sociedad?, sobre estas interrogantes, nos proponemos caracterizar las políticas públicas como proceso de concertación Estado-Sociedad, para ello se observaron los planteamientos de diversos autores respecto del análisis de políticas públicas, para establecer el contexto referencial a estudiar, sobre el cual extraer las conclusiones.

\section{Políticas Públicas}

El término políticas públicas (PP), se asocia de inmediato con asuntos del gobierno y sistemas políticos o como actividades de las instituciones públicas, que van dirigidas a tener una influencia determinada sobre la vida de los ciudadanos,

1 El autor define la política como aquellos programas de acción, lo que supone la elección específica de medios para obtener ciertos objetivos (Ballart, 1992: 37), y alega que un programa, basándose en Rutgnan (1984, citado por Ballart, 1992: 37), consiste en una intervención o en un conjunto de actividades desarrolladas para satisfacer alguna necesidad social reconocida o solucionar un problema identificado.

2 A los fines del presente artículo, nos referimos a las políticas sociales y abordamos su tratamiento como políticas públicas en general. Considerando las políticas sociales como aquellas, que responden a dificultades sociales, bien sea, modificar o solucionar situaciones adversas al bienestar social. 
para considerarse como pública tiene que haber sido generada, o al menos procesada en el marco de los procedimientos, instituciones y organizaciones gubernamentales.

Subirats (1989: 40), considera el término polisémico, bien sea, entendido en un campo de actividad (política social, política exterior); como medio para alcanzar fines (política a seguir en casos de conflicto); como conjunto de programas u objetivos que tiene el gobierno en un campo determinado (política energética); como resultado final, output o producto (el subsidio de paro, para paliar el desempleo); como out-come, impacto cierto sobre la realidad (política de vivienda); como proceso, secuencia de hechos y decisiones que buscan modificar una realidad (política sanitaria).
Esto, evidencia que la PP siempre se dirige a una situación que requiere una intervención por parte del Estado, para lograr ciertas transferencias directas o indirectas a la sociedad. Pueden incluir orientaciones o contenidos, instrumentos o mecanismos, así como aspectos institucionales. Sin embargo en algunas podría faltar uno o más de los elementos mencionados, cuyos objetivos pueden ser confusos o meramente declarativos.

En el Cuadro 1 se presentan las definiciones planteadas por distintos autores estudiados.

Puede observarse, que los autores tienen como elemento común la participación del Estado en la elaboración de PP para resolver problemas sociales, la refieren como actividades, cursos de acción, resultados, proposiciones o como transformaciones. No obstante, Lahera

\section{Cuadro 1}

\section{Comparación conceptual del término política pública}

\begin{tabular}{ll}
\hline Autor & Definición de política pública \\
\hline Rose (1984) & Transformación y combinación que hacen organizaciones gubernamentales \\
& de los recursos normativos, humanos, financieros y tecnológicos con el fin de \\
& atender los problemas de los ciudadanos, controlar sus comportamientos, sa- \\
& tisfacer sus demandas y, de esta manera, lograr impactos sociales, políticos y \\
& económicos.
\end{tabular}

Subirats (1989) Actividades de las instituciones de gobierno, actuando directamente o a través de agentes, y que van dirigidas a tener una influencia determinada sobre la vida de los ciudadanos.

Lahera (1999) Cursos de acción o flujos de información relativos a un objetivo público, desarrollados por el sector público con la frecuente participación de la comunidad o el sector privado.

Repetto (2000) Resultado de la interacción entre actores sociales y estatales, moldeados por marcos institucionales.

Piñango (2003) Proposiciones gubernamentales sobre la mejor forma de lograr determinados objetivos sociales. Ideas que contienen toda la fuerza y debilidades de éstas, no obstante las mejores ideas pueden generar efectos indeseables.

Fuente: Elaboración propia, 2006 
enfatiza la colaboración de la comunidad y del sector privado, y Repetto menciona actores sociales participantes en dicho proceso. En tal sentido, y tomando en consideración estos planteamientos, se propone a efectos de esta investigación la siguiente definición de política pública: Disposiciones del Estado para atender determinadas realidades que afectan directa o indirectamente a la sociedad, sean de tipo social, política o económica.

Pero, para que logren los resultados más cercanos a los óptimos, se requiere que se diseñen, ejecuten y evalúen en las mejores condiciones y con la mayor utilidad social, en tal sentido los diferentes autores opinan que; se requiere la acción de un buen gobierno (Lahera, 2000: 23), los grupos y sectores entran en la dialéctica del conflicto y la negociación, conformando coaliciones que apoyan la elaboración e instrumentación de determinada acción u obstaculizan una o ambas (Repetto, 2000: 601), se orientan hacia el progreso y bienestar de los ciudadanos (Piñango, 2003: 263, 266). En consecuencia, se podría deducir que las PP determinan el tipo de sociedad existente, en razón de que a través de ella se dictaminan las prestaciones (tributos, ayudas, servicios, beneficios, obligaciones) entre el Estado y la sociedad.

Se observa que desde su inicio responde a determinados objetivos, pero los mismos pueden modificarse en su trámite de elaboración, lo cual resulta comprensible en razón del largo y complejo proceso que involucra la creación de toda política pública de once (11) etapas conforme con Subirats (1989: 42), referidas al análisis de: 1) percepción y definición del problema, 2) intereses afectados, 3) grado de organiza- ción, 4) acceso a los canales representativos, 5) consecución del estatuto propio de tema a incluir en el programa o agenda de actuación de los poderes públicos, 6) formulación de una solución o de una acción de respuesta, 7) establecimiento de objetivos y prioridades, 8) soportes políticos presupuestarios y administrativos de la solución propuesta, 9) implementación o puesta en práctica de esa política, 10) evaluación y control de los efectos producidos y mantenimiento, 11) revisión o terminación de esa política.

Por su parte Rose (1984, citado por Vila y Chedrese, 2003: 10), plantea desde una perspectiva de análisis, que las PP pueden ser comprendidas como un proceso de cinco fases estrechamente relacionados entre sí: 1) identificación y definición del problema; 2) formulación de las alternativas de solución; 3) adopción de una alternativa; 4) implementación de la alternativa seleccionada y 5) evaluación de los resultados obtenidos.

A partir de los planteamientos de Subirats y Rose, se reconoce analíticamente que las PP se conciben como un conjunto de fases, para Subirats sumamente complejo y detallado, que implica un proceso complicado y extenso, por el contrario para Rose resulta una secuencia racional apropiada, pero pareciera que relega la evaluación solo a los resultados, sin estimar las otras fases del proceso.

De la confrontación de ambas propuestas, se sugiere un procedimiento de formación de políticas conformado por seis (6) fases: 1) reconocimiento del problema e inclusión en la agenda del gobierno, 2) posibles soluciones al problema, 3) aceptación y reconocimiento de la propuesta de solución, 4) implementa- 
ción de la propuesta y su evaluación, 5) seguimiento y evaluación de resultados, 6) seguimiento y evaluación del impacto de la política.

A continuación se presenta el Diagrama 1, el cual permite apreciar como de entrada la política, ofrece solución a los problemas que dejados a la acción individual no hallan salida, también facilita procesos de identidad colectiva al considerar una estrecha interrelación entre actores sociales y políticos en cada una de sus fases, en razón que, las acciones enfrentadas por los gobiernos no solo dependen de las decisiones por parte de los funcionarios públicos, sino más bien del resultado de las interacciones entre éstos y los actores interesados, que bien pudiera referirse a lo que Subirats (1989: 129) denomina como intereses afectados.
En todo caso, la PP se relaciona con una variedad de circunstancias, personas, grupos y organizaciones, de los cuales depende su formulación y puesta en práctica. Estos factores determinan un escenario complejo y heterogéneo, que se complica al entrar en escena un elemento axiomático la cuestión social, que relaciona régimen político, Estado y sociedad, de cuyo acuerdo o convergencia dependen plenamente la elaboración de políticas públicas sociales de calidad, favorables al colectivo.

\section{La Política Pública Social: dimensión social del Estado}

Este grupo de políticas están dirigidas a la satisfacción de las necesidades básicas de la población, para lo cual el es-

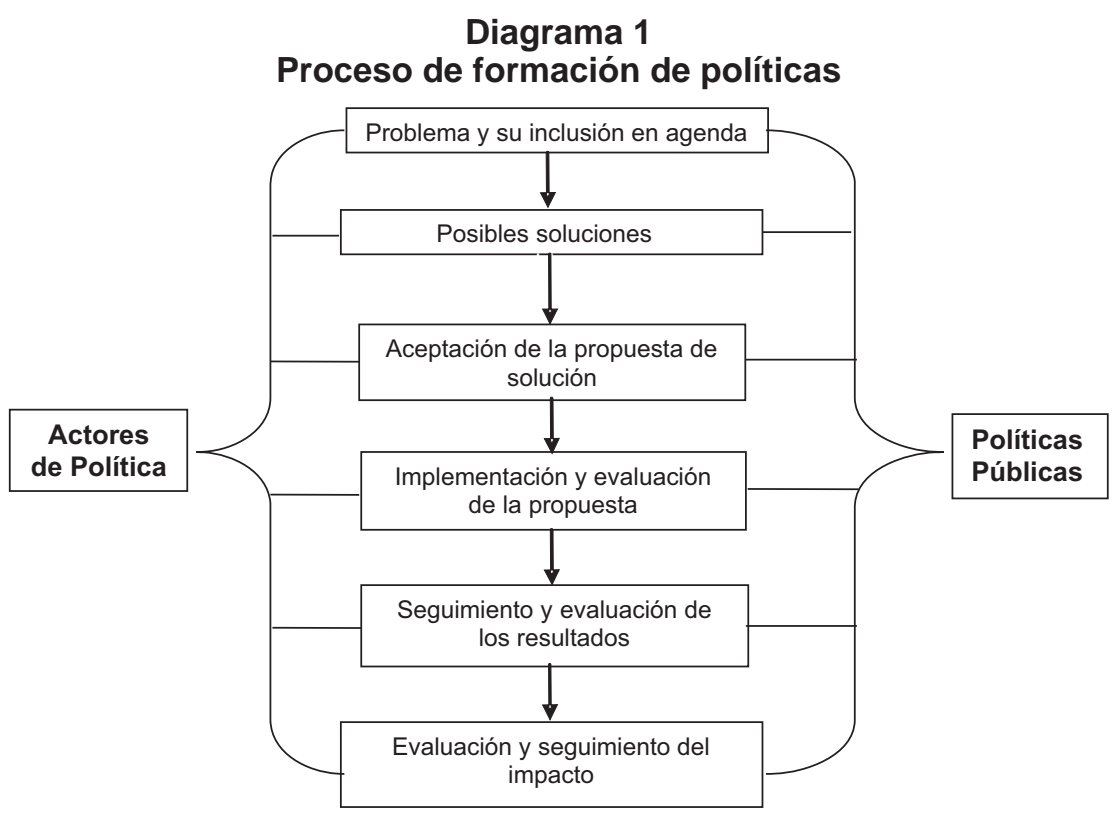

Fuente: Elaboración propia, 2006, con base en Subirats y Rose. 
tado resulta fundamental, esto fue dejado en claro por los autores examinados en el punto anterior en su enfoque social de la política (Rose, 1984; Subirats 1989; Lahera, 2000; Repetto, 2000 y Piñango, 2003), al plantear enfáticamente que toda PP debe tener una dimensión social como principal orientación.

En la gestión de las políticas sociales, la jerarquía del Estado es indiscutible, perfectamente reconocido por el Centro Latinoamericano para el Desarrollo (CLAD), cuando expone "el Estado continúa siendo un instrumento fundamental para el desarrollo económico, político y social de cualquier país" (1998: 7), toda política, deriva de la decisión y acción pública, en la cual la actuación de los personajes e instituciones involucrados adquiere características particulares, por un lado, la puesta en práctica de estas políticas calibra a través del tema social la relación entre gobierno, Estado y sociedad, y por otro plasma la dificultad participativa de los beneficiarios al no lograr constituirse en protagonista del proceso, en la definición de criterios importantes en torno a éstas, como su cobertura y alcance, y las orientaciones expuestas a continuación.

Sagasti y otros (1999: 13, 14), en un análisis sobre la política social en América Latina, la consideran como: el conjunto de pronunciamientos, intervenciones y acciones gubernamentales conducentes al bienestar de los individuos, las familias y los grupos sociales, asumiendo un enfoque caracterizado por:

1. Equidad, un nivel de vida mínimo compatible con la dignidad humana, y el pleno desarrollo de las personas, como nuevo enfoque de política social.
2. Interacciones entre lo social-políticoeconómico para integrar plenamente a la política social con las otras políticas de desarrollo.

3. Diferenciar criterios tales como nivel de aplicación (nacional regional, local) y según los sujetos de las medidas de política (género, edad, etnia, ingresos).

4. Considerar explícitamente las dimensiones internacionales de la política social, para una mayor equidad global y un entorno adecuado para la política social regional y nacional.

5. Adoptar una actitud pluralista y de experimentación social, que convierta el diseño y ejecución de políticas sociales en un proceso de aprendizaje colectivo.

De acuerdo con estos autores, la política social contiene fundamentalmente tres conjuntos de acciones por parte del gobierno:

- Provisión de servicios sociales básicos (educación, salud, nutrición, saneamiento, seguridad social).

- Lucha contra la pobreza

- Generación de empleos

En este contexto de análisis, a continuación se presenta un cuadro resumen del concepto de política social y sus diversos objetivos.

El Cuadro 2 muestra que las concepciones de Valadez y Bamberger, Sagasti y Delgado tienen como fortaleza el rol dominante del Estado en cuanto a estructura institucional-organizativa y disposición de recursos financieros, pero al mismo tiempo una fragilidad del lado de los ciudadanos, en detrimento de los beneficiarios, al decidir el Estado sobre los criterios de los servicios y cobertura, a 


\section{Cuadro 2}

Conceptualización y objetivos de la Política Social

\begin{tabular}{|c|c|c|}
\hline Autor & Política Social & Objetivo \\
\hline $\begin{array}{l}\text { Valadez y Bamberger, } \\
1994\end{array}$ & $\begin{array}{l}\text { Programas diseñados por el Estado } \\
\text { para mejorar la calidad de vida por me- } \\
\text { dio del mejoramiento de la capacidad de } \\
\text { los ciudadanos para participar, en acti- } \\
\text { vidades sociales, económicas y políti- } \\
\text { cas, tanto a nivel local como nacional }\end{array}$ & Mejorar la calidad de vida \\
\hline $\begin{array}{l}\text { Sagasti y otros, } \\
1999\end{array}$ & $\begin{array}{l}\text { Pronunciamientos, intervenciones y ac- } \\
\text { ciones gubernamentales orientados a } \\
\text { aumentar el bienestar de los individuos, } \\
\text { las familias y los grupos sociales }\end{array}$ & $\begin{array}{l}\text { Bienestar de los individuos, las } \\
\text { familias y los grupos sociales }\end{array}$ \\
\hline $\begin{array}{l}\text { Garretón, } \\
2001\end{array}$ & $\begin{array}{l}\text { Programas para producir sociedad, } \\
\text { igualdad socio-económica y calidad de } \\
\text { vida, ciudadanía y actores sociales fuer- } \\
\text { tes y autónomos que negocien su repre- } \\
\text { sentación en las esferas políticas y es- } \\
\text { tatales }\end{array}$ & $\begin{array}{l}\text { Igualdad socio-económica y } \\
\text { calidad de vida }\end{array}$ \\
\hline $\begin{array}{l}\text { Delgado, } \\
2001\end{array}$ & $\begin{array}{l}\text { Conjunto de objetivos, regulaciones, } \\
\text { sistemas y entidades por medio de los } \\
\text { cuales el Estado se dirige a crear opor- } \\
\text { tunidades y fortalecer instrumentos en } \\
\text { términos de la equidad y la integración } \\
\text { social. }\end{array}$ & Equidad e integración social \\
\hline
\end{tabular}

Fuente: Elaboración propia, 2006.

través de los programas, de modo que los usuarios no tienen elección, no obstante podrian generar espacios, mediante la participación ciudadana, que contribuyan a que los derechos y deberes institucionalmente establecidos sean efectivamente reconocidos, permitiendo un verdadero intercambio entre la sociedad civil y el poder estatal.

Por su parte Garretón presenta como debilidad que parte de suponer la igualdad socio-económica, calidad de vida, ciudadanía y actores sociales fuertes como elementos presentes en la sociedad y con capacidad de negociación, pero este contexto dista mucho del existente en América Latina, donde de acuerdo con el CLAD (1998: 8), problemas estructurales como: consolidación de la democracia, crecimiento económico y desigualdad social, ocupan aún la atención del Estado. Así mismo, Valadez y Bamberger, Sagasti, Delgado y Garretón comparten como objetivo de la política social, la calidad de vida, ya sea en forma explicita o implícita.

A los efectos de esta investigación, y partiendo de la definición teórico conceptual de los autores antes analizados, se propone la siguiente definición de polí- 
tica social: conjunto de pronunciamientos y acciones en forma de programas, formulados por el Estado, en convergencia con las directrices de desarrollo local, regional y nacional, dirigidos a mejorar el bienestar social. Esta concepción responde a un razonamiento reflexivo de la naturaleza social, política y económica de toda PP, sobre la base de que éstas, se identifican como toda forma de acción organizada que busca objetivos de interés común.

Este escenario refleja lo que para Delgado (2002: 117) serían las transferencias del Estado hacia la sociedad, bien sea, en forma de recursos financieros, servicios de salud, educación, seguridad social, seguridad alimentaria, vivienda, protección laboral y asistencia a las familias, en función de derechos, leyes y políticas de acción positiva. Garretón (2001, citado por Delgado, 2002), argumenta que la política social, desde una perspectiva de justicia, participa de una doble dinámica. Primero, se espera que materialice los derechos del bienestar, mejorando la calidad de vida y facilite una ciudadanía activa y productiva; segundo, que intervenga directamente en la generación de reglas de juego e instituciones que permitan el ejercicio de los derechos civiles y políticos para aquellos que son excluidos de la toma de decisiones.

Esta última apreciación, contiene elementos implícitos como la relación Es- tado y sociedad a través de los efectos esperados de la política, con especial referencia a la ciudadanía activa, capaz de inducir espacios plurales para la toma de decisiones, donde los actores participantes expresen, argumenten y cuestionen las diferentes propuestas y decisiones, de modo que se logre favorecer una acción de interés público ${ }^{3}$ que sobreponga el beneficio colectivo sobre el político.

\section{Actores de política: Estado y Sociedad}

En la actualidad, la dinámica social demanda una PP que opere para reconstruir la conexión Estado-Sociedad a través de la generación de espacios, vínculos, sentido de pertenencia y bienestar para la población. Para esto, es propicio un ambiente tendente a la implementación de determinados mecanismos de participación directa de los actores afectados que refuercen, por un lado, la función receptiva de la sociedad y, por el otro, la capacidad utilitaria del Estado.

Para comprender el entramado de los actores de política social, bajo la premisa de que las decisiones estatales expresen las necesidades del conjunto de la sociedad, se requiere del concurso de la democracia, que garantice la actuación de una ciudadanía capaz de intervenir activamente en las decisiones, compartidas con los representantes del Estado, des-

3 Existe una íntima relación entre la opinión pública y la dinámica política, expone el debate público como la única posibilidad de superar los conflictos sociales, gracias a la búsqueda de consensos que permitan el acuerdo y la cooperación pese a los disensos (Habermas, 1982, en Forum Barcelona, 2004). 
virtuados en la práctica concreta de su función social. En consecuencia los actores de política desarrollan sus guiones de acuerdo a su escenario estatal o social

Se aclara brevemente la acepción sobre Estado, Gobierno y Sociedad, los cuales guardan intrínseca relación. El fenómeno Estado, es la organización política más importante dentro de la sociedad (Loaiza, 2004: 55). Independientemente del tipo de Estado, la sociedad que le sirve de base o la ideología predominante, en la práctica el poder reside en el gobiemo "conformado por un grupo de individuos que comparten una responsabilidad dada en el ejercicio del poder político del Estado" (Loaiza, 2004: 106), en el entendimiento de que ejercen una autoridad legítima y que, mediante unas decisiones que toman protegen y dirigen la comunidad.

Para el CLAD (1998: 8) "el papel del Estado debe orientarse primordialmente hacia el área social, asegurando la universalidad de los servicios de salud, y educación y hacia la creación de instrumentos que ayuden a promover el desarrollo económico". Pero si bien es cierto que salud y educación, están entendidamente relacionados con desarrollo económico, estos no constituyen prioridades a lograr en la sociedad venezolana por parte del Estado dirigente, no obstante aquella no muestra intransigencia sobre tales prestaciones, será que desconocen la universalidad de los mismos o la carencia de ellos les impide actuar.

Por otra parte, el hombre adquiere una personalidad social a través de un proceso de adaptación y comunicación simbólica asumiendo unos roles que lo capacitan para vincularse activamente al fenómeno llamado sociedad, para lo cual se precisan ciertos requisitos que aseguren la permanencia dentro de ésta, como la motivación suficiente, mediante las posibilidades de desarrollo personal, logro de objetivos sociales y limitaciones a todo acto pernicioso, así como el manejo de pautas de comportamiento (Loaiza, 2004: 96, 97), en la realidad representan necesidades que de no estar resueltas, deberían constituir el objeto de la acción del Estado.

La ilustración de estos términos, evidencia el entablado de la escena de los actores de política pública, quienes protagonizan los complejos procesos para el diseño e implementación de éstas, donde es significativa la capacidad de presión de algunos, mediante el empleo de los recursos de que dispone para conseguir sus fines.

De acuerdo con lo anterior, podemos distinguir en general dos categorías de actores: sociales y políticos, apoyándonos en Pírez (1995: 3, 4) los actores sociales son unidades reales de acción en la sociedad, tomadores y ejecutores de decisiones que inciden en la realidad social de la cual forman parte, actúan como individuos o colectivos, que están sometidos a ciertas condiciones culturales, étnico-culturales, políticas y territoriales. Los actores políticos reproducen su poder en relación a los otros actores y en referencia al gobierno, son unidades abiertas que pueden ser penetradas por la sociedad, sin la cual no sobrevivirian, tal es el caso de políticos que sin los votos o sin el apoyo de ciertos grupos dejarían de serlo.

A tal efecto, los actores sociales se consolidan mediante organizaciones no gubernamentales (ONG), sindicatos, 
consejos comunales y toda forma de organización que agrupe a la sociedad civil, de forma que si la actuación del Estado es permeable a la opinión social, se garantiza una mayor transparencia a la actuación gubernamental, al tiempo que se incrementa la confianza de la ciudadanía.

Pero, no se debe identificar todo colectivo con un actor relevante, bien sea por parte del Estado como de la sociedad, se requieren ciertas condiciones que lo definan como tal. Al respecto, Repetto y Acuña (2001, citados por Vila y Chedrese, 2003: 11) estiman la presencia de ciertos atributos en determinados colectivos o individuos, que los califica como actores de política pública:

1. Capacidad de negociación: este atributo da cuenta de la posibilidad de incidir en el proceso de política pública a partir de la movilización de los recursos políticos y económicos que se posean. Aun cuando estos autores no plantean de forma explícita el significado de capacidad de negociación, este podría entenderse en términos de Porter (1980: 66), como la habilidad o suficiencia de alguno de los actores de influir en el proceso de creación de una política.

2. Capacidad para descifrar el contexto: Se refiere a la posibilidad que se tenga de acceso a la información relevante para poder influenciar en las diferentes instancias.

3. Capacidad de representación: Alude a la posibilidad de contar con la legiti- midad $y / o$ legalidad necesarias para actuar en nombre de otros.

4. Capacidad de movilización social: Lo que importa aquí es contar con un número significativo de adherentes plausibles de ser movilizados en un momento dado, como medio de presión.

5. Capacidad de acción colectiva: En el caso de actores colectivos, estos elevan sus reales posibilidades de influencia en la medida que logran controlar el problema del free rider ${ }^{4}$. En lo que se refiere a los actores estatales, estos estarían en condiciones de movilizar un recurso que les es propio, la capacidad de autoridad (posibilidad de ejercer el poder público en función de que, en última instancia, las políticas son fijadas e implementadas por el Estado).

Tal como quedo manifiesto, los actores del Estado disponen del atributo de autoridad, lo que les confiere cierta posición. Se percibe como, estos atributos permiten que los grupos e individuos dispongan de recursos para participar en las instancias claves del ciclo de las políticas, por supuesto esta dinámica depende del sistema institucional vigente.

El Diagrama 2 permite establecer en relación con los actores de política que una vez que se definen, en función de las potencialidades que tengan o puedan adquirir, la forma como éstos interactúan incide en las capacidades estatales de resolución de los problemas sociales. Este 


\section{Diagrama 2 Actores de Política Pública}

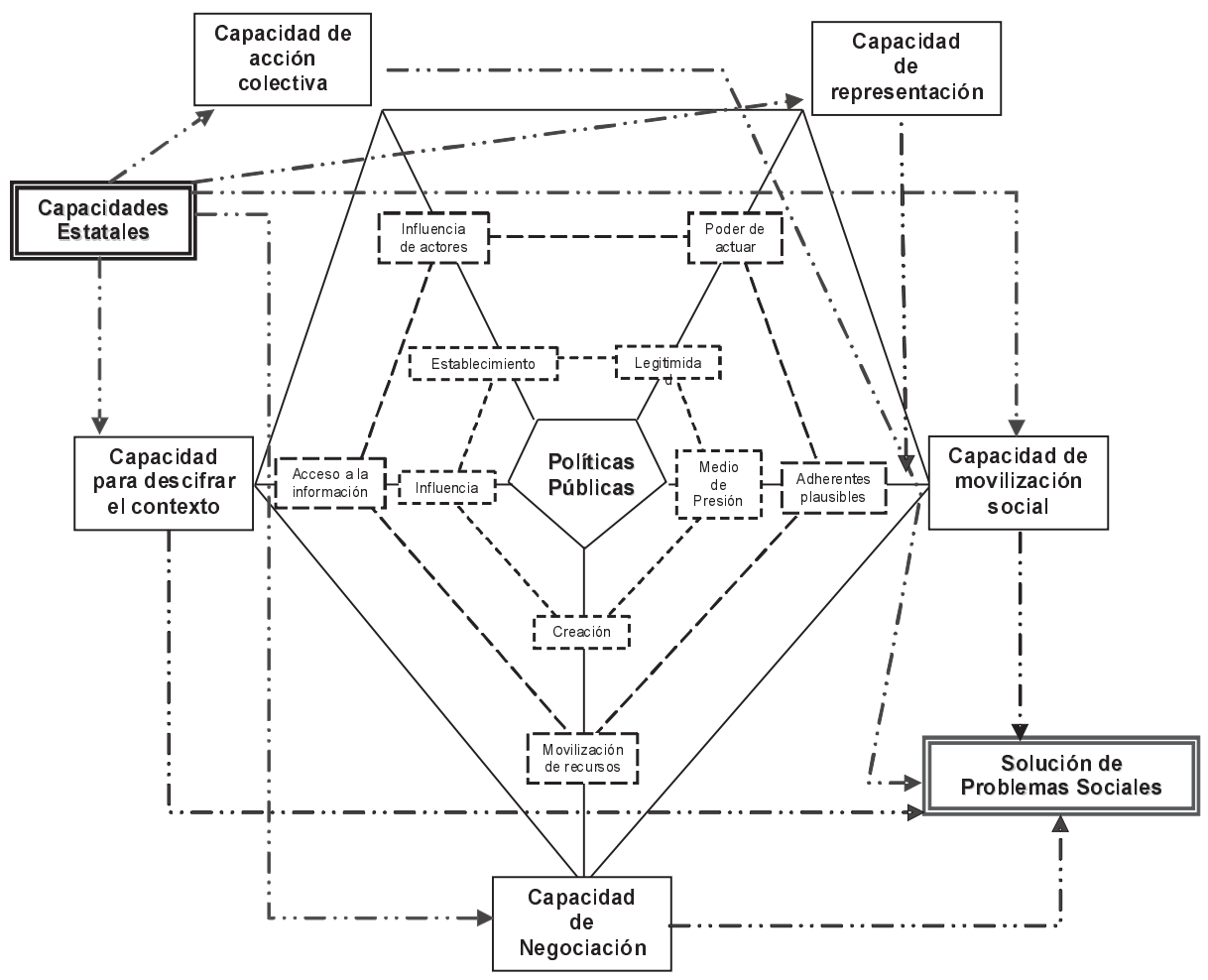

Fuente: Elaboración propia 2006, basado en Repetto y Acuña (2001).

contexto permite visualizar la estructura de poder que pudiera ejercer dominio en el proceso de la elaboración de una política y cuáles actores impondrían sus intereses en la agenda de gobierno.

Es en este entorno donde los gobernantes pretenden hacer valer su posición frente a las cuestiones reconocidas como problemas por los actores sociales. En otras palabras, los actores pueden intervenir estratégicamente en la vida pública a través de las políticas en función de sus intereses reales para resolver los problemas de la sociedad. Tal vez, esto explica por qué no siempre encontramos una coherencia entre la definición del problema y las opciones de solución propuestas.

"El proceso de decisiones, especialmente en sistemas democráticos, obedece a una dinámica política compleja, en la cual entran en juego los intereses de los sectores dominantes, las presiones de diversos actores, resistencias burocráticas y finalmente condiciones objetivas que obligan a decisiones que mu- 
chas veces nada tienen que ver con lo planificado o con la voluntad de quienes formalmente dirigen el aparato estatal" (Ochoa et al., 1999: 47-48). En fin, la decisión de resolución, mejoramiento o prevención de determinada situación o complicación, resultaría de la interacción entre los actores sociales y estatales, los cuales, a través de ajustes recíprocos, argumentaciones, relaciones de poder, información, conocimiento, destrezas y mediaciones profesionales, construyen y estructuran una propuesta aceptable, que alcanza consenso dentro de una perspectiva real, de modo que se de la eficiente intervención pública.

En este sentido, si entendemos lo público como el punto de encuentro de los intereses colectivos, es fundamental contar con la participación responsable de la sociedad civil como elemento de confrontación/acuerdo en cada una de las etapas de los programas públicos que se implementen (diagnóstico, diseño, ejecución, evaluación y control), de manera que las organizaciones que conforman esta sociedad civil, contraparte privilegiada en el seguimiento de las políticas sociales, dada su proximidad a la ejecución de las mismas, resulta un componente evaluador de la actuación pública.

En nuestro caso, el Estado venezolano ha reglamentado esta participación, mediante la Ley de los Consejos Comunales (Asamblea Nacional, 2006: 3) y la
Constitución Bolivariana vigente (1999), expone lineamientos que propician la participación social, como son los controles sociales y participación ciudadana4 en la gestión pública (artículos 62, 70-72).

Bajo este modelo plural de participación ciudadana ${ }^{5}$, en el sentido que se propicia la actuación del colectivo, a través de las diferentes organizaciones civiles o de ciertas capacidades, antes mencionadas, presentes en los actores sociales y estatales. La observación del accionar de éstos actores principales, permite comprender la relación entre el Estado y la sociedad y la normativa por la cual se rigen, que la mayoría de las veces cruza una línea que separa lo acordado y lo que se hace.

En suma, el consenso requiere la confluencia de diversos elementos que conformarian un espacio válido para el debate; la democracia, una sociedad participativa, un Estado eficiente y canales de participación efectiva, para lograr una auténtica relación entre el Estado y la sociedad civil.

Para analizar las políticas públicas es necesario considerar la presencia de actores políticos y sociales, partir del análisis de los actores en cada una de las fases del proceso de políticas, con el propósito de vislumbrar el mapa de actores que interactúan se podría mejorar y fortalecer el sistema de evaluación de las inversiones públicas, para aumentar la eficacia

5 La participación ciudadana puede ser entendida como un medio de fortalecimiento de la sociedad civil, porque le incorpora vigencia, le da viabilidad, es el instrumento de aproximación a las decisiones públicas (Araya, 2005, citado por Mendoza, 2006: 4). 
del gasto social y su gestión, al logro de los objetivos que persiguen, generalmente el bienestar social, lo que a su vez sería el soporte que justifique la renovación o extensión de políticas y/o programas públicos.

\section{Evaluación de Políticas Públicas}

En la actualidad, la necesidad de diseñar políticas exitosas para mejorar las condiciones de vida de la población, demandan la cooperación del Estado y la sociedad, y de la responsabilidad y transparencia del desempeño público, para alcanzar beneficios en primera instancia sociales y consecuentemente económicos y culturales, vía para ganar la confianza y respeto de la sociedad.

Desde esta perspectiva, algunas sociedades poseen mecanismos de aprendizaje político y social que, desde el gobierno como en el caso de los Estados Unidos, o desde el control social como en algunos países europeos, favorecen la decisión sobre políticas públicas, la rendición de cuentas y el conocimiento de la ciudadanía sobre las acciones de sus gobernantes.

Claro que, la capacidad estatal define quiénes estructuran y llevan adelante las políticas, con qué medios cuentan para hacerlo y bajo qué reglas de juego operan. Tal como lo expone Repetto (2001: 53), la acción del estado, no deriva solamente de sus características endógenas, sino también de la estructura institucional, política y socioeconómica, no obstante los procesos de la Reforma del Estado, han modificado la relación y articulación del Estado con la sociedad, en las etapas de definición del problema, implementación y evaluación de la política.

Para que una política logre sus objetivos, requiere del engranaje de ciertos factores, a lo largo de todo su proceso, estos factores de acuerdo con Vila y Chedrese (2003: 23) son: 1. Grado de profesionalización de la administración, 2. Estilo de gestión política y 3 . Características de los actores involucrados (grado de participación de la sociedad), pero lo fundamental es la forma en que estos tres factores armonicen. Reconocen, los autores el ámbito local (municipios) el lugar por excelencia para innovar, a través de la implementación de mecanismos de participación que permitan estrechar la relación Estado/Sociedad, condición sine qua non para la relegitimación del sistema político municipal.

Dentro de esta temática, la evaluación en el campo político y social, constituye un efectivo instrumento de aprendizaje para la ciudadanía, asi como una herramienta ${ }^{6}$ de transformación de la administración pública, útil para diseñar o modificar políticas, particularmente aquellas orientadas al mejoramiento de las condiciones de vida de la población, ya que al analizar la acción del Estado, se puede

La evaluación como herramienta referencial para conocer y tomar decisiones sobre los aspectos que están condicionando la marcha sobre los aspectos de la Administración Pública y herramienta para racionalizar la toma de decisiones (Osuma, 2002: 49). 
según los resultados obtenidos cuestionar o acordar su acción.

La evaluación de políticas, programas y proyectos sociales "es una actividad programada de reflexión sobre la acción, basada en procedimientos sistemáticos de recolección, análisis e interpretación de información, con la finalidad de emitir juicios valorativos fundamentados y comunicables, sobre actividades, resultados e impactos de esos proyectos o programas y formular recomendaciones para tomar decisiones que permitan ajustar la acción presente y mejorar la acción futura" (Nirenberg et al., 2000: 32).

De acuerdo con las anteriores apreciaciones la evaluación, es un proceso transversal a toda la política, implica una definición del propósito, carácter, metodología, instrumentación y análisis de resultados, pero con la particularidad de que aún cuando es parte de este asunto, constituye un elemento de juicio que le permite a los gobiernos aprender sobre las consecuencias de sus acciones, para poder decidir, sobre cierta política, a través de los organismos de control nacionales, regionales o locales, igualmente los organismos civiles a favor de los intereses del colectivo.

Así mismo, contribuye al mejoramiento de la administración pública, no solo en función de los resultados obtenidos, sino mediante el mejoramiento o modificación de la formulación, el diseño y puesta en práctica de sus políticas, en este caso el Estado es co-actor de la resolución de los problemas colectivos, ya que a la Sociedad le corresponde el papel estelar. Al respecto, Subirats (1995: 76) considera la evaluación un proceso global y realista que implica interacción del evaluador con los protagonistas de la implementación de las políticas y programas, dificultándose una separación estricta entre las evaluaciones y la política, pero encuentra como mayor problema, no el reducir el contenido político de las evaluaciones, sino cómo evitar reducir el proceso de evaluación a un mero ritual destinado a ratificar o atacar una política particular

Se podría afirmar, que la evaluación sobre políticas y programas sociales, es un proceso que va más allá de analizar los aspectos burocráticos formales, de gerencia tradicional o la forma en que actúa el aparato público, en sentido amplio y genérico, incluye la evaluación de políticas, planes, programas, proyectos, servicios, instituciones y materiales. Sobre esto, Weiss $(1997: 19,20)$ aclara que se desarrolla en un escenario de acción, destacando que el objetivo de la evaluación es describir, entender relaciones entre variables y seguir la secuencia causal de una variable con otra. Por lo tanto, la evaluación pudiera considerarse como un recurso al servicio de la sociedad, que apunta a incorporar, con todo lo problemático que esto puede resultar, a los destinatarios de los programas.

Estas consideraciones teóricas, facilitan observar la evaluación como un instrumento favorecedor para el Estado al mejorar el desempeño administrativo con transparencia, donde los actores evaluadores interactúan, en función de sus intereses o los que representan, dado el caso que la sociedad logre participar en tal proceso evaluativo. Sobre este análisis Jon-Sung y Khagram, indican que "en sociedades muy polarizadas por la desigualdad, amplios sectores tienen limitada 
información, poca capacidad de organización, y son débiles para monitorear a los grupos minoritarios que concentran los ingresos. En las elites a su vez, este cuadro de poder concentrado con poco control social, puede generar incentivos hacia prácticas corruptas porque crea una situación de cuasi-impunidad" (2004, citados por Kliksberg, 2006: 7).

Una forma de evitar estas prácticas corruptas es asegurar, la transparencia, a través de la participación ciudadana, a quien le corresponde exigir la practica de la rendición de cuentas por parte del Estado, tanto la una como la otra deben establecer una perfecta simbiosis en pro de asegurar una gestión pública sana a los ojos de la sociedad. En palabras de Guerrero:

"La transparencia y la rendición de cuentas están aliadas, pero no deben confundirse. El gobierno democrático debe rendir cuentas para reportar o explicar sus acciones. $Y$ debe transparentarse para mostrar su funcionamiento y ser sujeto de escrutinio público. Con la rendición de cuentas nos aseguramos que las autoridades se responsabilicen ante alguien de sus actos. Con la transparencia exigimos a los funcionarios que actúen según principios admitidos por todos, pues frecuentemente la necesidad de transparentar no es otra cosa sino la necesidad de contar con el concurso y la aprobación del público" (2003, citado por Mendoza, 2006: 5).

Es el caso que la rendición de cuentas por parte del Estado, al poner en conocimiento de la sociedad cuestiones fundamentales sobre los pormenores de la administración público, desencadena procesos de aprendizaje, pues la sociedad procurará información, capacitación, organización, pero solo y solo si logra percibir que su participación puede desencadenar cambios favorables, de modo que sirvan de motivación para su participación.

La evaluación es vista por varios autores entre ellos, Subirats (1995), Weiss (1997), Repetto (2001), Niremberg (2000) y Vila y Chedresse (2003), como un proceso de análisis destinado a producir información válida sobre el desempeño de una política y que esta información serviría para la toma de decisiones en cuanto a: modificar, continuar o eliminar una política y/o programa público. En razón de ello emitir un juicio acerca de una política, deriva en una lógica de conflicto y acuerdo, elementos presentes en todo proceso de evaluación, por otra parte la externalidad que hasta ahora ha caracterizado a la evaluación de PP, ha contribuido a agudizar el conflicto que dificulta su ejecución, planteándose la incertidumbre sobre la posibilidad de convertir las evaluaciones en prácticas organizacionales normales y efectivas, en todo proceso de PP, para Ballart (1992: 72) evaluar supone la idea de juzgar el mérito, el valor o la bondad de las políticas y de los programas públicos..

En el Diagrama 3 expuesto a continuación, se evidencia la relación directa entre los resultados de una política (productos, efectos directos e impactos) y la toma de decisiones con relación a ésta, respecto de su continuidad, posible modificación o eliminación por parte de los actores de política. 


\section{Diagrama 3 \\ La evaluación en el análisis de una Política}

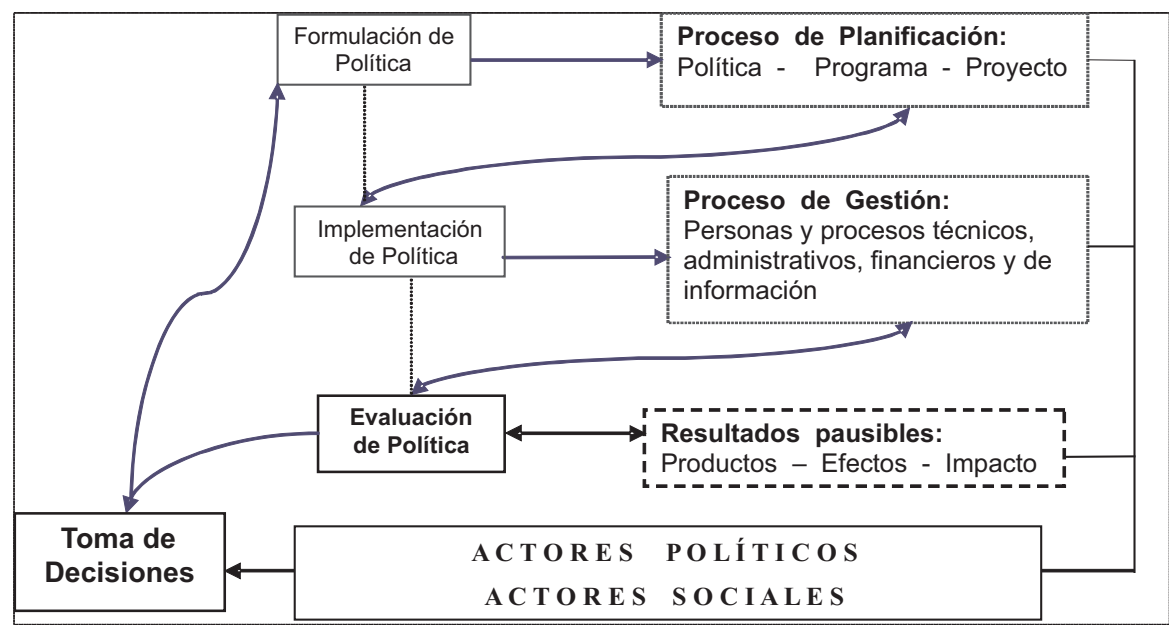

Elaboración Propia, adaptado de Rossi y Freeman (1985, citado por Ballart, 1992).

En definitiva lo importante es reconocer la función de la evaluación para la sociedad, al inducir el aprendizaje en cuanto a las formas que tiene el Estado para responder a los desafíos sociales que impone el desarrollo, este aprendizaje al mismo tiempo traduce el logro de políticas y programas sustentables en el tiempo con la finalidad de alcanzar el máximo beneficio y participación de los ciudadanos en procesos plurales, transparentes y efectivos, que al fin de cuentas deberían contar con el apoyo concreto y palpable del Estado.

En contraparte de esto, la evaluación promueve controversias del siguiente orden:

- Relaciones tensas entre responsables de políticas y evaluadores.

- Discordancias, entre la acción de los políticos, la percepción del problema por la comunidad y el interés del evaluador.
- Dificultad para ajustar los resultados con la pluralidad de destinatarios e intereses comprometidos.

Además la falta de correspondencia entre el discurso y la práctica de las evaluaciones y la no utilización de los pocos resultados obtenidos, al menos en el ámbito nacional, reprime la función de la evaluación de las políticas sociales para construir sociedad, fortalecer actores sociales, reforzar vínculos y colaborar con una convivencia cívica y democrática que propicie la concertación Estado/Sociedad. En consecuencia, la práctica de la evaluación en la gestión pública, con pluralidad de actores comprometidos e interesados en sus resultados, facilitaría la participación ciudadana, que en suma representa el escenario óptimo para el establecimiento de la articulación Estado/Sociedad. 


\section{Conclusiones}

Se indican las derivaciones del análisis de los planteamientos teóricoconceptuales sobre políticas públicas, políticas sociales, actores de política y evaluación de políticas públicas, en el marco de las relaciones entre el Estado y la sociedad a lo largo del proceso de las políticas públicas, a manera de deducciones finales.

El proceso de formación de una política pública resulta problemático, y en el caso de las sociales siempre responde a demandas sociales, bien sea una necesidad o un problema de la colectividad, cuyas fases desde su inclusión en agenda hasta su evaluación se relaciona con actores plurales (estatales y sociales), los cuales influyen en la toma de decisiones, mediante la retroalimentación del proceso. De modo que para que un problema social (salud, analfabetismo, alimentación, vivienda, servicio de agua, violencia familiar), logre mutar hacia un problema político es necesario que los ciudadanos organizados o cierto profesional clave, logren desarrollar, algunas capacidades que les permita transformarse en actores de política.

Estos actores sociales, con sus necesidades y aspiraciones al lado de los intereses y presiones por partes de los actores estatales, inician una confrontación hasta lograr negociar el problema e incorporarlo en la agenda de gobierno, pero aquí no termina esto, una vez encontradas alternativas de solución, resultado también de una disputa entre actores interesados, estos nudos críticos del proceso de políticas explica por qué no siempre encontramos consistencia entre la situa- ción problema y las opciones de solución. El caso es que no siempre las personas más cercanas al problema hacen valer sus criterios en las acciones que resultan respaldadas por parte del gobierno, como políticas públicas.

A pesar, de los logros por parte de la sociedad, en las últimas décadas, para que sus necesidades sean escuchadas e incorporadas en las agendas gubernamentales, las características conflictivas y subjetivas del proceso, resaltan la dificultad de hacer políticas. Precisamente, parte de esta complicación viene dada por la interacción establecida por los intereses del Estado y por las necesidades de la sociedad, así como por los marcos institucionales (normativas, reglamentaciones, otras leyes) que moldean la acción de ambos, no obstante debe reconocerse que la acción de la sociedad por si sola no es determinante, para la solución de sus necesidades y problemas, de modo que precisa del apoyo del Estado para tal fin.

Surge obligatoriamente esta interrogante, cómo asegurar que una vez que se ha logrado la decisión estatal, ésta se traduzca en transferencias reales y plausibles hacia la sociedad, que aseguren mejores niveles de calidad de vida, aquí también la sociedad, juega un papel insustituible exigiendo y participando en la evaluación de las políticas implementadas y solicitando la rendición de cuentas, sobre la gestión de las políticas sociales, por parte de los funcionarios públicos responsables de ésta.

Otro aspecto importante, además de la transparencia por parte del Estado y de la participación ciudadana, es la innovación gubernamental, fundamentada en 
las tecnologías modernas, tanto organizacionales como administrativas y un capital humano, profesional y capacitado, de modo que se logre conformar una administración pública honesta, equitativa, que rinda cuentas, en fin que se rija bajo la cultura de la eficiencia y beneficio social. No cabe duda, en esta dimensión moderna de la gestión pública, del rol protagónico del Estado, al lado de una sociedad emprendedora.

Pero, lo más relevante en la concertación Estado-Sociedad, serían los conocimientos, habilidades y experiencias adquiridos por los ciudadanos, que permitirian un aprendizaje colectivo, y consecuentemente un empoderamiento social útil, condición sine qua non para construir el espacio Estado-sociedad óptimo, en estas condiciones aún ventajosas para el Estado, se vislumbra el principio de los valores que catapultan un Estado fuerte, eficiente y confiable y una sociedad, informada, participativa y activa.

En este ámbito político, la parte que toca promocionar es la de la sociedad ya que la del Estado está asegurada por la naturaleza propia del proceso de PP, lo argumentado en los párrafos anteriores, nos indica que la construcción de una cultura cívica, apuntala el seguimiento de la acción gubernamental, mediante la participación social y la rendición de cuentas.
Podría decirse que la participación social, en la experiencia nacional, permanece "in freezing state", en los actuales momentos, se espera que con la puesta en práctica de la Ley de los Consejos Comunales, la participación se haga activa en pro del bienestar social.

Sobre la base de la evaluación de políticas públicas, éstas distan mucho de ser imparciales, generalmente, las mismas políticas y/o programas públicos establecen los criterios para su evaluación en el caso de que la considere, asimismo son llevadas a cabo por agencias o personal designado por la administración pública, que pone en duda la objetividad de la misma. Sin embargo en caso de obviar la neutralidad y relación con el gobierno de los evaluadores, ellos tienen sus inclinaciones políticas, su ideología y cultura que de acuerdo con su profesionalismo, sesgaría los resultados de tal evaluación.

Bajo esta premisa se podría especular que en función de las derivaciones de esta fase del proceso de PP, sería prudente una tendencia pluralista ${ }^{7}$, multiactoral orientada a la utilización ${ }^{8}$, para que en ella estén representados todos los actores intervinientes, los estatales y los diversos actores sociales incluyendo los beneficiarios de la política y/o programa público. Esto, acredita que a medida que se afinan los puntos de interés para los

7 Se centra en la importancia de los valores y de las opiniones de los diferentes actores que tienen que ver con un programa público. Reconoce la naturaleza política de la evaluación (Ballart, 1992: 153).

8 Para que la evaluación constituya un proceso orientado a la utilización/relevancia social, deberían asegurarse a todos los participantes, efectivas oportunidades de intervenir desde la fase de selección del objeto de la evaluación. 
actores y la utilidad de lo obtenido, simultáneamente, se precisen los diferentes canales políticos y administrativos a los cuales compete la evaluación de determinada política social.

En fin, se requiere del concurso de la sociedad organizada que actuando desde el nivel municipal asuma el seguimiento de las políticas sociales, en función de su proximidad a la ejecución de las mismas e interés en solucionar sus problemas. Desarrollando, instancias de intercambio viables y sencillas hacia las autoridades locales, regionales y nacionales, de modo que se legitime y enriquezca la actuación de cada uno de los participantes en las políticas públicas, mediante una estrecha relación entre el Estado y la sociedad.

\section{Referencias Bibliográficas}

Asamblea Nacional de la República Bolivariana de Venezuela (2006). Ley de los Consejos Comunales. Gaceta Oficial $N^{\circ}$ 5.806. Caracas, Venezuela.

Asamblea Nacional Constituyente (1999). Constitución de la República Bolivariana de Venezuela. Caracas.

Ballart, Xavier (1992). ¿Cómo evaluar programas y servicios públicos? Aproximación sistemática y estudios de caso. 1ra. Ed. Madrid, España. Editorial Gráficas Baneth SA. 284 p.

Cardozo, Mirian (2003). Evaluación de políticas de desarrollo social. En: Revista Política y Cultura. $N^{\circ} 20$, pp. $139-$ 154.

CLAD (1998). Una Nueva Gestión Pública para América Latina. Caracas, Venezuela. $24 \mathrm{p}$.

Delgado, Adriana (2001). Formulación participativa de políticas sociales en la ges- tión distrital y local. Resultados parciales de la validación del diseño metodológico en Bogotá. En: Revista del CLAD, N²1, pp. 116-142.

Forum Barcelona (2004). Historia y crítica de la opinión pública. Recuperado el 1405-200, desde: http://www.barcelona2004.org/esp/actualidad/biblioteca/biblioteca

García, Néstor (2000). "Políticas culturales en tiempos de globalización”. En: Revista de Estudios Sociales, N5. Bogotá, pp. 19 - 35.

Kliksberg, Bernardo (2005). ¿Cómo avanzar la participación en América Latina, el continente más desigual? Recuperado el 14-05-2007, desde: http://cemupro.com.ar/biblioteca. php?pos $=5 \&$ orden_modo=DESC \& criterio $=$

Loaiza, Hernando (2004). Estado, Gobierno y Gerencia Pública. 1ra. Edición. Bogotá, Colombia. Publicado por Universidad Santo Tomás. 318 p.

Mendoza, Jorge (2006). Bases conceptuales, doctrinarias y jurídicas de la figura de los testigos sociales. $\mathrm{XI}$ Congreso Internacional del CLAD sobre la Reforma del Estado y de la Administración Pública, Guatemala, 7 10 Nov. Recuperado el 14-05-2007, desde: http://www.clad.org.ve/fulltext/ 0055215.pdf

Nirenberg, O. Brawerman, J y Ruiz, V. (2000). Evaluar para la transformación: innovaciones en la evaluación de programas y proyectos sociales. Buenos Aires. Argentina. Editorial Paidós, Colección Temas Sociales.

Ochoa, H. López, M. y Rodríguez, I. (1996). Administración pública y populismo en Venezuela. En: Revista Venezolana de Gerencia, Vol. 1, N 1. Maracaibo, pp. 39 - 58.

Osuma, José (2002). La Evaluación participativa: una visión integral y pluralista. 
En Banón Rafael y Martínez (Comp). La evaluación de la acción y de las Políticas Públicas. Madrid, España. Ediciones Díaz de Santos, 280 p.

Piñango, Ramón (2003). Políticas públicas en América Latina. Teoría y práctica pp. 249-277. 1ra. Ed. Caracas, Venezuela. Ediciones IESA, $287 \mathrm{p}$.

Pírez, Pedro (1995). Actores sociales y gestión de la ciudad. En: Ciudades 28, Octubre-Diciembre. México, $12 \mathrm{p}$.

Porter, Michael (1982). Estrategia competitiva. Técnicas para el análisis de los sectores industriales y de la competencia. Editorial Continental, México (versión original editada en 1980), 407 p.

Repetto, Fabián (2001). Gestión pública y desarrollo social en los noventa: Las trayectorias de Argentina y Chile. 1ra. Ed. Buenos Aires, Argentina. Editorial Prometeo, $336 \mathrm{p}$.

Sagasti, F. Iguiñiz, J. y Schudt J. (1999). Equidad, Integración Social y Desarrollo: hacia un nuevo enfoque para la política social en América Latina. 1ra. Ed. Perú. Ediciones Universidad del Pacífico. Centro de investigaciones, $147 \mathrm{p}$.

Subirats, Joan (1989). Análisis de Políticas Públicas y Eficacia de la Administración. 1ra. Ed. Madrid, España. Editorial Imprenta Nacional del Boletín del Estado, $184 \mathrm{p}$.

Valadez, J. y Bamberger, M. (1994), Policymakers, Managers and Researchers. Recuperado el 14-4-2005, desde: http://www.preval.org/documentos/ 00430.pdf.

Vila, María y Chedrese, Lucas (2003). Los Consejos Barriales: propuesta para el fortalecimiento democrático. Repensando la participación política desde lo local. Ponencia en II Congreso Argentino de Administración Pública. Sociedad, Gobierno y Administración. Recuperado el 29-12-2005, desde: http://www.aaeap.org.ar/ponencias/ congreso2/Vila-Chedrese.pdf

Weiss, Carol (1997). Investigación Evaluativa. Métodos para determinar la eficacia de los programas de acción. 4ta. Reimpresión. México. Editorial Trillas. $183 \mathrm{p}$. 